\title{
Some mathematical tools for modelling malaria: a subjective survey
}

\author{
Jacek Banasiak $^{1,2}$, Rachid Ouifki ${ }^{1}$, Woldegebriel Assefa Woldegerima ${ }^{1}$ \\ ${ }^{1}$ Department of Mathematics and Applied Mathematics \\ University of Pretoria, South Africa \\ ${ }^{2}$ Institute of Mathematics, Łódź University of Technology, Poland \\ Correspondence: wa.woldergerima@up.ac.za, assefa@aims-cameroon.org
}

Received: 9 June 2021, accepted: 2 October 2021, published: 13 October 2021

\begin{abstract}
In this paper, we provide a brief survey of mathematical modelling of malaria and how it is used to understand the transmission and progression of the disease and design strategies for its control to support public health interventions and decisionmaking. We discuss some of the past and present contributions of mathematical modelling of malaria, including the recent development of modelling the transmission-blocking drugs. We also comment on the complexity of the malaria dynamics and, in particular, on its multiscale character with its challenges and opportunities. We illustrate the discussion by presenting a curve fitting using a $95 \%$ confidence interval for the South African data for malaria from the years 2001-2018 and provide projections for the number of malaria cases and deaths up to the year 2025.
\end{abstract}

Keywords: Mathematical modelling, malaria, South Africa, data fitting

\section{Malaria AND its mathematical Modelling}

Malaria is an indirectly transmitted disease of humans that requires the interaction of three dis- tinct living organisms (the components) to sustain transmission. These are:

1) Parasites of genus Plasmodium that causes malaria disease in humans. Four species of Plasmodium have long been recognized to infect humans and cause illness, namely, $P$. falciparum, P. malariae, $P$. vivax and $P$. ovale. The fifth one, $P$. knowlesi, that naturally infects macaques have recently been recognized to cause zoonotic malaria also in humans, but no cases of the humanmosquito-human transmission have been reported. Of these species, $P$. falciparum is responsible for most malaria deaths globally and is the most prevalent species in SubSaharan Africa, [77]. P. vivax is the second most significant species and is prevalent in Southeast Asia and Latin America. P. vivax and $P$. ovale have the added complication of the dormant liver stage which, after some time, can be reactivated in the absence of a

Copyright: (C) 2021 Banasiak et al. This article is distributed under the terms of the Creative Commons Attribution License (CC BY 4.0), which permits unrestricted use, distribution, and reproduction in any medium, provided the original author and source are credited.

Citation: Jacek Banasiak, Rachid Ouifki, Woldegebriel Assefa Woldegerima, Some mathematical tools for modelling malaria: a subjective survey, Biomath 10 (2021), 2110029, 
J Banasiak, R Ouifki, W A Woldegerima, Some mathematical tools for modelling malaria: a subjective ...

mosquito bite, leading to clinical infection, [29], [77].

2) Humans (host), where parasites grow and multiply first in the liver cells and then in the red cells of the blood, producing merozoites which cause symptoms and illness, and gametocytes which is the form of the parasites that can be transferred to the mosquito during a blood meal.

3) Female Anopheles mosquitoes (vector), which are the agents responsible for transmitting the disease from one human to another. They become infected when they feed and ingest human blood that contains mature gametocytes. After a mosquito picks up the gametocytes, they start another cycle of growth and multiplication in the mosquito, eventually producing sporozoites. When the mosquito takes a blood meal on another human, it injects the sporozoites with its saliva and infects humans. Thus, the mosquito carries the parasite from one human to another, acting as a vector.

Despite malaria being preventable and treatable, it remains one of the most prevalent and deadliest human infection in developing countries, especially in the sub-Saharan Africa, where young children and pregnant women are most affected, [78]. According to the latest WHO malaria report, released on the 30 November 2020, there were 229 million cases of malaria in 2019 compared to 228 million cases in 2018. The estimated number of malaria deaths stood at 409000 in 2019, while there were 405000 deaths in 2018. The WHO African Region carries a disproportionately high share of the global malaria burden with as much as $94 \%$ of malaria cases and malaria deaths recorded there in 2019, [77]. It is feared that the number of malaria deaths may increase in the years 2020/2021 due to the COVID-19 pandemic, especially in the sub-Saharan Africa, where the number of COVID-19 cases is rising, [69]. First, malaria and COVID-19 share several common symptoms such as fever, breathing difficulties, tiredness or acute headache, which may lead to misdiagnosis, particularly when the clinicians rely mainly on the symptoms, [43]. Further, COVID-19 can disturb and affect the control and treatment of malaria in different ways including delays in the distribution of the insecticide-treated bed nets (ITNs), difficulties in testing and treatment in hospitals over-crowded with COVID-19 patients and due to restricted availability of the health workers.

Fighting malaria requires coordinated research across many disciplines, with mathematics and mathematical modelling playing an important role. Here we use mathematical tools to represent and analyze real-world processes to make predictions or otherwise provide insights about their dynamics. In mathematical epidemiology, we create simplified representations, called models, of diseases such as malaria in a population, to understand how the infection may progress in the future. Mathematical models are the cheapest and often the only way to test different scenarios for the development of a disease and various interventions such as vaccination programs, [1], [16], [52]. Thus, they are crucial to study the malaria transmission mechanism and the dynamics of its progression, predict and estimate the prevalence and incidence and evaluate strategies for control. Hence, they help to inform the public health interventions policy-decision making. For instance, results obtained from mathematical models may predict which populations are most vulnerable to the disease, allowing for focusing antimalarial drugs and preventative treatments on high-risk groups. However, the effectiveness of such models and their robustness largely depend on the choice of model and the researcher's adherence to the assumptions governing the chosen model's use.

Over the past century, many mathematical models of various complexity levels, some discussed in more detail in Section 2, have been developed. They have been used to answer the following questions related to its development, growth, progression and transmission in endemic and high transmission areas. 
J Banasiak, R Ouifki, W A Woldegerima, Some mathematical tools for modelling malaria: a subjective ...

1) What are the predicted peak number of cases and the time of its occurrence? What is the expected cumulative number of cases over the epidemic (i.e. final attack rate)?

2) What is the basic reproduction number $\left(\mathcal{R}_{0}\right)$ and the current value, or the time course, of the effective reproduction number?

3) What might be/is the potential impact of antimalarial drugs?

4) How should antimalarial drugs be prioritized for the distribution among the population subgroups to minimize infectiousness for those infected, prevent the development of resistance, and shorten the duration of the illness?

5) When should the vaccination be introduced, even though there is no currently approved vaccine for malaria? What would be the impact of vaccine timing on the benefits of vaccination? Can the vaccine/drug effectiveness be predicted?

Mathematical analysis can be applied at various levels, such as the disease transmission dynamics between humans and mosquitoes, the in-host immuno-pathogenesis dynamics of the malaria parasites, or pharmacokinetics (PK) and pharmacodynamics (PD) properties of the drugs and vaccines. We emphasize here that though there is no currently approved vaccine for malaria, the search for malaria vaccine continues and mathematical models can help in the theoretical design, clinical trials, and the vaccine's deployment. Withinhost modelling of infectious diseases has drawn significant attention in the last half-century due to its significance in improving our understanding of how the microscopic processes develop and affect the host health. Besides, it is crucial to understand how the within-host dynamical processes (immunological processes) of the parasite impact on the population-level dynamics of the disease spread (epidemiological processes), [53]. In this paper, however, we will focus on the populationlevel compartmental malaria transmission models.

\section{A BRIEF REVIEW OF MATHEMATICAL MODELS OF} MALARIA

\section{A. History and background of mathematical mod-} els of malaria

Mathematical epidemiology (modelling of infectious diseases) can be traced back to the work of Daniel Bernoulli. He formulated and solved a model for smallpox in 1760 and used it to evaluate the effectiveness of the inoculation of healthy people against the smallpox virus, [1]. Much later, William H. Hamer [36] formulated and analyzed a model to understand the recurrence of measles epidemics. He was the first to propose that the spread of infection should depend on the numbers of susceptible and infective individuals and suggested the mass action law for the rate of new infections. His ideas have served as a foundation of compartmental models since then, [15]. In 1927 William O. Kermack and Anderson G. McKendrick, [44], developed a general epidemic model and described the relationship between susceptible, infected and immune individuals in a population. The Kermack-McKendrick epidemic model has successfully predicted the behaviour of outbreaks in many epidemics, [15], [45].

Mathematical modelling of malaria dates back to the work of Sir Ronald Ross. He was the first to understand malaria's human-mosquito transmission mechanism, winning for this achievement the Nobel Prize in Medicine in 1902. Subsequently, in 1911 he developed a compartmental differential equation model of malaria as a host-vector disease, [67]. Ross divided the human and mosquito populations into the susceptible (S) and infectious (I) classes and used the so-called $S_{h} I_{h} S_{h}$ model for humans and $S_{v} I_{v}$ for mosquitoes, where $S_{h}, I_{h}, S_{h}$ and $S_{v}, I_{v}$ represent susceptibles and infectious humans and mosquitoes, respectively. Using his model, he showed that reducing the mosquito population below a critical level would be sufficient for malaria elimination. In other words, contrary to the common belief of that time, there is no need to kill all mosquitoes to control the disease. This result has been since known as the mosquito theorem. 
J Banasiak, R Ouifki, W A Woldegerima, Some mathematical tools for modelling malaria: a subjective ...

Ross' model was extended in 1957 by George Macdonald, who included the exposed (infected but not infectious) mosquito class, [50]. The RossMacdonald model of malaria transmission has had a significant influence on malaria control. One of its main conclusions is that malaria's endemicity is most sensitive to the changes in the mosquito survival rate. The Ross-Macdonald model allows for several other conclusions. First, it shows that malaria can persist in a population only if the number of mosquitoes is greater than a given threshold. Second, the prevalence of infections in humans and mosquitos depend directly on the basic reproductive number calculated in the paper. The basic reproduction number, usually denoted by $\mathcal{R}_{0}$, is defined as the average number of infections produced by one infectious individual introduced into a fully susceptible population over the duration of the infectious period, [26], [73]. It depends on the duration of the infectious period, the probability of infecting a susceptible individual upon contact, and the number of new susceptibles contacted in a unit of time, i.e., the number of newly infected individuals per unit time, [39], [40]. In the malaria context, $\mathcal{R}_{0}$ should take into account that an infectious human must first infect a mosquito, which then generates secondary infections among humans, thus determining it is not straightforward. $\mathcal{R}_{0}$ helps determine whether an infectious disease can spread through a population. In particular, if $\mathcal{R}_{0}<1$, then each infectious individual produces on average less than one new infectious individual, and thus the disease should die out in the long run. On the other hand, if $\mathcal{R}_{0}>1$, then each infectious individual infects on average more than one individual, and thus the infection should be able to spread, [26], [73].

It is worth noting that the researchers such as R.A. Ross, W.H. Hamer, A.G. McKendrick or W.O. Kermack who, between 1900 and 1935, laid the foundations of the mathematical approach to epidemiology, were not mathematicians but public health physicians, [15]. This observation emphasizes the fact that though biological and epidemiological research has greatly improved our knowledge of the life cycle of the malaria parasite within humans and mosquitoes, it cannot achieve a complete understanding of the complexities of malaria on its own. Hence, resorting to mathematical models that can integrate various multiscale and intertwined aspects of the disease and, by using mathematical tools such as local and global stability analysis, bifurcation analysis, sensitivity analysis and data fitting, yield short- and longterm predictions about its progress, proves to be a necessity. The models, however, should reflect the current epidemiological knowledge and thus, they evolve to incorporate the latest empirical findings. To wit, the Ross-Macdonald model assumed that infected humans cannot develop immunity against malaria and that the human and mosquito populations are homogeneous. With the evidence showing that this was an oversimplification, the model has been extended in different directions by incorporating acquired immunity, variability in the mosquito and human populations, demography and age-structure, the environment or other relevant factors such as more realistic transmission mechanisms. We briefly mention some of these extensions. In [7], the authors considered only the human population but also incorporated the class of immunized humans and included an age structure. The work in [4] included the exposed classes and introduced a $S_{h} E_{h} I_{h} S_{h}$ and $S_{v} E_{v} I_{v}$ compartment model for humans and mosquitoes, respectively. (Ngwa \& Shu, 2000) [57] also study an $S_{h} E_{h} I_{h} S_{h}$ and $S_{v} E_{v} I_{v}$ compartment model but considering varying population sizes. In [20], the authors extended the latter by including constant human immigration. An important addition to the malaria transmission models was the inclusion of the immunity function to the human population in [27]. Socioeconomic factors in the human population and environmental factors in the mosquito population have been considered in [83] and others. In [58], the authors formulated a mathematical model that incorporated one aquatic stage of the mosquitos and availability of the adult vector and human treatment. They introduced, in particular, the so-called basic offspring number whose 
magnitude determines the existence of a thriving mosquito population in the sense that when this number is below unity, then the mosquito population becomes extinct. In recent years, the climate change has attracted attention in the field, and this resulted in a series of papers considering this aspect in modelling, see, e.g. [31], [62], [61], [56], [83]. Other malaria transmission models incorporate treatment in their models, see, e.g. [24], [60]. It is reassuring that in [32], the authors used current modelling techniques to show that there is no bistable equilibrium in the malaria transmission and therefore, that elimination is feasible.

Mathematical modelling of malaria can also include information about how different parts of the population, such as adults and children, interact, and how these interactions influence the infection's spread, resulting in age-structured mathematical models, where the hosts are grouped into compartments composed of individuals in the same age-group and infection status, see e.g., [12], [28], [34], [33], [37]. Age-structured models are important to address intervention strategies involving the treatment with novel antimalarial drugs and vaccines. In [34] the authors fitted real data to an age-structured model to investigate the effectiveness of intervention strategies in reducing malaria parasites' count. In the subsequent paper, [33], an age-structured model was used to estimate the changing age-burden of $P$. falciparum malaria using real data in sub-Saharan Africa.

It is important to note that the process of complete eradication of an infectious disease such as malaria is divided into five main phases, defined in [55] and summarized in [76, p.2]. These are the transmission control, the disease elimination, the infection elimination, the eradication and the extinction. Mathematical modelling of malaria can be applied at each of these phases. Several models, including the ones discussed above, were developed for the phases "the transmission control" or "the disease elimination ". Some recently published mathematical models considered the other phases. For example, (White, et al., 2009), the authors used a mathematical model that required the input data in the form of a single estimate of parasite prevalence to consider "the infection elimination" phase. Their model included critical interventions targeting malaria transmission, which are currently available or in the final development stages. Their results showed that a simple model has a similar short-term dynamic behaviour to complex models. They also demonstrated that the population level protective effect of multiple controls was crucial to overcoming failed elimination attempts. However, it is important to realize that even though a simple mathematical model could be suitable for situations where the data are sparse, more complex models, populated with new data, would provide more information, especially in the longterm, [75].

A recent development in new anti-malaria drug design opens new avenues for mathematical modelling. For instance, in [17] the authors estimated the transmission reduction that can be achieved by using drugs of varying chemo-prophylactic or transmission-blocking activity and they concluded that transmission reductions and eradication of malaria depend strongly on the deployment strategy, treatment coverage and endemicity level. In [80], the authors formulated a mathematical model that incorporates the effects of transmissionblocking drugs (TBDs) on reducing the number of malaria infections. Our mathematical results predict, in particular, that treating with TBDs both clinical and subclinical malaria infections is required malaria eradication.

\section{B. Different types of mathematical models in malaria}

Generally, several types of mathematical models have been used to describe the transmission dynamics of infectious diseases, such as malaria. These are the deterministic models, stochastic models, statistical models, computer-based models, etc. Several modelling approaches consist in using the available data to derive the process's statistical parameters and a fitted curve describing its dynamics. We refer to them as statistical modelling; they can be used mainly for predictions, information extraction, and description 
of stochastic structures, see [46], [22]. However, statistic modelling mostly reveals correlations and not causations in the studied process. For that we need mechanistic models which attempt to reflect the causal relations in the process and thus, by using appropriate conservation laws and constitutive relations, give a description of the process's dynamics, based on the understanding of its driving mechanisms. In deterministic models of this kind, the model's output is fully determined by the parameter values and the initial conditions, without any room for random variation or uncertainty. Deterministic models with continuous time can be written using ordinary, partial, or delay differential equations, [1], [16], while if we use discrete time intervals, the model takes the form of a difference equation, see [48] for an example of a discrete malaria model.

On the other hand, while recognizing and using the causal relations in the described process, stochastic models allow for randomness in one or more inputs, making the evolution not precisely predictable. This implies that the same set of parameter values and initial conditions lead to an ensemble of different outputs, [1], [16] and thus the outcome can be predicted only with some probability. Some authors have used a stochastic process to model malaria, e.g. [47], [54], [64], [11], [35], just to mention a few.

Since mathematical models are approximations of real-life phenomena, each type of mathematical model has its advantages and disadvantages. We have listed some of the limitations of mathematical models in the conclusion section, Section 6 . However, we emphasize that the models based on our understanding of the mechanisms driving the described processes tend to have better potential for making robust and reliable predictions.

Though we recognize that for successful control and eradication of malaria it may be necessary to use a combination of different mathematical modelling approaches [2], [51], in this paper, we shall focus on deterministic compartmental models discussed above and discuss some of them in more detail. Such models, consisting of appropriately constructed systems of ordinary differential equations, are the most used mathematical tools in modelling malaria transmission dynamics.

\section{GENERIC MATHEMATICAL MODEL OF MALARIA}

In this section, we present a generic malaria model and explain how to use it to represent the disease's complex transmission dynamics.

Mathematical modelling of malaria begins with collecting and understanding basic biological facts relevant to the disease. These facts form the model's assumptions and rephrased in mathematical terms, become its constitutive relations. The model then consists of an appropriate system of conservation laws with the coupling described by these constitutive relations.

For instance, in a population of humans of size $N_{h}$ in a region affected by malaria, we can distinguish a group of individuals who are not infected by the malaria parasites but are at risk of getting it; they are called susceptibles and denoted by $S_{h}$. The remaining individuals, who are infected and can pass the infection to others are called infectious and denoted by $I_{h}$. Individuals in this compartment may fail to recover and die or can recover (either due to natural causes or medication), and then move to the recovered, $R_{h}$, group. However, it is known that recovering from malaria induces only temporary immunity, so, after a certain time, the recovered can get reinfected upon being bitten by an infectious mosquito. Such individuals will move from the $R_{h}$ class back to the $S_{h}$ class. We can refine the description of this process by introducing the exposed class $E_{h}$ of infected but not infectious humans to account for the malaria incubation time. Models describing such a progression of malaria in humans are called $S_{h} I_{h} R_{h} S_{h}$ or $S_{h} E_{h} I_{h} R_{h} S_{h}$ models, respectively. Analogous models can be constructed for the mosquito population.

The progression between the compartments is a conservative process in the sense that the in- 


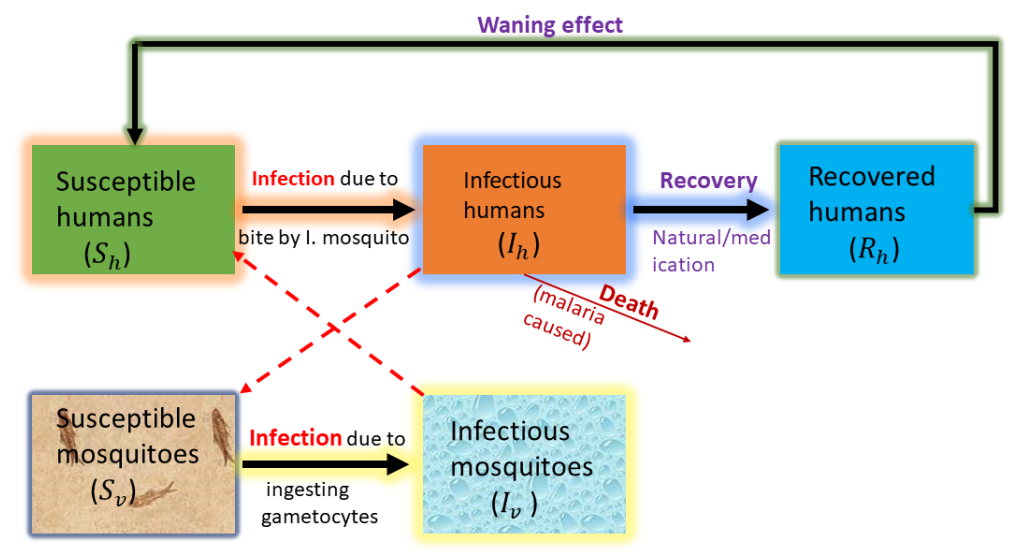

(a) Simple SIR-SI flow diagram for transmission dynamics of malaria.

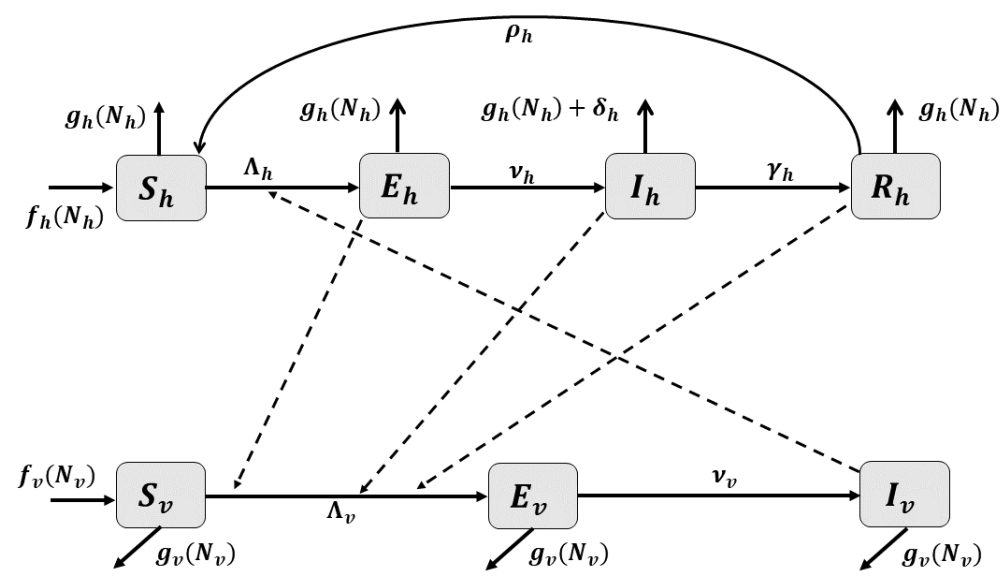

(b) Flow diagram for $S_{h} E_{h} I_{h} R_{h} S_{h}-S_{v} E_{v} I_{v}$ transmission dynamics of malaria.

Figure 1: Simple SIR-SI flow diagram (a) and a flow diagram for $S_{h} E_{h} I_{h} R_{h} S_{h}-S_{v} E_{v} I_{v}$ (b) transmission dynamics of malaria.

dividuals in, say, the class $S_{h}$ that become infected, vanish from this class but must reappear in the infectious class. The mechanism of the progression is modelled then by appropriate constitutive relations. We must rephrase these notions using mathematical functions, expressions and equations. Usually, the first step in constructing a model is representing the relevant processes in a flow diagram. The diagram in Figure $1 \mathrm{a}$ shows the basic structure of such $S_{h} I_{h} R_{h} S_{h}-S_{v} I_{v}$ model for human-mosquito transmission dynamics of malaria, while Figure $1 \mathrm{~b}$ shows a flow diagram for an $S_{h} E_{h} I_{h} R_{h} S_{h}-S_{v} E_{h} I_{v}$ malaria model with demographic phenomena such as natural births and deaths, see, e.g.

A typical example of a mathematical $S_{h} E_{h} I_{h} R_{h} S_{h}-S_{v} E_{v} I_{v}$ model for transmission dynamics of malaria with demography, see, e.g. 
J Banasiak, R Ouifki, W A Woldegerima, Some mathematical tools for modelling malaria: a subjective ...

[20], [23], [57], [80], is given by

$$
\begin{aligned}
& \frac{d S_{h}}{d t}=f_{h}\left(N_{h}\right)+\rho_{h} R_{h}-\Lambda_{h} S_{h}-g_{h}\left(N_{h}\right) S_{h}, \\
& \frac{d E_{h}}{d t}=\Lambda_{h} S_{h}-\left(v_{h}+g_{h}\left(N_{h}\right)\right) E_{h}, \\
& \frac{d I_{h}}{d t}=v_{h} E_{h}-\left(\delta_{h}+\gamma_{h}+g_{h}\left(N_{h}\right)\right) I_{h}, \\
& \frac{d R_{h}}{d t}=\gamma_{h} I_{h}-\left(\rho_{h}+g_{h}\left(N_{h}\right)\right) R_{h}, \\
& \frac{d S_{v}}{d t}=f_{v}\left(N_{v}\right)-\Lambda_{v} S_{v}-g_{v}\left(N_{v}\right) S_{v}, \\
& \frac{d E_{v}}{d t}=\Lambda_{v} S_{v}-\left(v_{v}+g_{v}\left(N_{v}\right)\right) E_{v}, \\
& \frac{d I_{v}}{d t}=v_{v} E_{v}-g_{v}\left(N_{v}\right) I_{v},
\end{aligned}
$$

where $\Lambda_{h}$ and $\Lambda_{v}$ the forces of infections (infection rates) of humans and vectors, respectively and the total populations of humans and vectors at any time $t \geq 0$ is, respectively, $N_{h}(t)=S_{h}(t)+E_{h}(t)+$ $I_{h}(t)+R_{h}(t)$ and $N_{v}(t)=S_{v}(t)+E_{v}(t)+I_{v}(t)$.

\section{A. Types of the force of infection}

When an infectious female Anopheles mosquito bites a susceptible human, there is a probability that the parasite (in the form of sporozoites) will be injected into the human's bloodstream and travel into liver cells to infect hepatocytes. The process when a susceptible human gets infected by an infectious mosquito is represented in the model by a function called the force of infection (of humans), denoted here by $\Lambda_{h}$. The analogous process for the female mosquitoes when, after biting an infections human, the gametocytes form of the malaria parasite enters the mosquito's midgut, is represented by a function called the force of infection of mosquitoes, denoted here by $\Lambda_{v}$. The form of the force of infection has a significant impact on the dynamics of malaria, but it depends on many factors and should be carefully chosen for the problem. We shall briefly discuss the most common choices.

We begin with a general form of the force of infection introduced in [20] without clear justification. Here we shall derive this form based on the Holling type argument, (Holling, 1959). We begin with some introductory observations. The force of infection of humans, $\Lambda_{h}$, is the product of the number of mosquito bites a human can have per unit of time, $b_{h}\left(N_{h}, N_{v}\right)$, the probability of the transmission of the disease from the mosquito to human, $\beta_{h v}$ and the probability that the biting mosquito is infected, $\frac{I_{v}}{N_{v}}$. Similarly, the force of infection of vectors, $\Lambda_{v}$, is the product of the number of bites a susceptible mosquito can make per unit time on humans, $b_{v}\left(N_{h}, N_{v}\right)$, the probability of the transmission from an infectious human to vector and the probability that the bitten human is infectious, $\beta_{v h} \frac{I_{h}}{N_{h}}+\widetilde{\beta_{v h}} \frac{R_{h}}{N_{h}}$. Here we take into account that both $I_{h}$ and $R_{h}$ humans can be infectious with possibly different transmission probabilities, $0 \leq \widetilde{\beta_{v h}}<\beta_{v h}<1$. Therefore, the forces of infections of humans and vectors, respectively, are given by

$$
\begin{gathered}
\Lambda_{h}=b_{h}\left(N_{h}, N_{v}\right) \beta_{h v} \frac{I_{v}}{N_{v}}, \\
\Lambda_{v}=b_{v}\left(N_{h}, N_{v}\right)\left(\beta_{v h} \frac{I_{h}}{N_{h}}+\widetilde{\beta_{v h}} \frac{R_{h}}{N_{h}}\right) .
\end{gathered}
$$

It remains to derive the formula for the total number of bites per unit time. It can be written as

$$
b\left(N_{h}, N_{v}\right)=b_{h}\left(N_{h}, N_{v}\right) N_{h}=b_{v}\left(N_{h}, N_{v}\right) N_{v} .
$$

To proceed, we define two parameters, $\sigma_{h}$ and $\sigma_{v}$, that are, respectively, the constant average number of mosquito bites a human can receive (respectively, a mosquito can make on a human) per unit time. $\sigma_{h}$ depends on the human's exposed area, awareness, etc., while $\sigma_{v}$ depends on the mosquito gonotrophic cycle, its preference for human blood and the time used for feeding. Consider a certain period $T$ that, for a mosquito, can be split as $T=T_{n a}+T_{a}$, where $T_{n a}$ is the time where the mosquito cannot bite and $T_{a}$ is the time available for biting. Thus, in time $T$, the total number of bites received by all humans can be written as

$$
b\left(N_{h}, N_{v}\right) T=\sigma_{h} T_{a} N_{h} .
$$

Now, if a mosquito can make only $\sigma_{v}$ bites in a unit time, it means that it is not able to bite 
J Banasiak, R Ouifki, W A Woldegerima, Some mathematical tools for modelling malaria: a subjective ...

for $1 / \sigma_{v}$ after each bite. Besides, in time $T$ it has $\frac{\sigma_{h} N_{h} T_{a}}{N_{v}}$ meals. Hence

$$
T=T_{a}+\frac{\sigma_{h} N_{h} T_{a}}{N_{v} \sigma_{v}} .
$$

Thus, plugging (3) into (2) and simplifying yields

$$
b\left(N_{h}, N_{v}\right)=\frac{\sigma_{v} \sigma_{h} N_{v} N_{h}}{\sigma_{v} N_{v}+\sigma_{h} N_{h}} .
$$

Therefore, the force of infections are modelled by

$$
\begin{gathered}
\Lambda_{h}=\frac{\sigma_{v} \sigma_{h}}{\sigma_{v} N_{v}+\sigma_{h} N_{h}} \beta_{h v} I_{v}, \\
\Lambda_{v}=\frac{\sigma_{v} \sigma_{h}}{\sigma_{v} N_{v}+\sigma_{h} N_{h}}\left(\beta_{v h} I_{h}+\widetilde{\beta_{v h}} R_{h}\right) .
\end{gathered}
$$

As noted in [20], these formulae generalize several previously used expressions for the forces of infection. In particular, if $N_{v} / N_{h}$ is small, then factoring out $N_{h}$ from the denominator and setting $N_{v} / N_{h}$ to 0 , we obtain

$$
\Lambda_{h}=\sigma_{v} \beta_{h v} \frac{I_{v}}{N_{h}}, \quad \Lambda_{v}=\sigma_{v} \frac{\beta_{v h} I_{h}+\widetilde{\beta_{v h}} R_{h}}{N_{h}},
$$

which are known as standard infection forces, (Anderson, 2013), (Allen, 2008), (Martcheva, 2015), (Hethcote, 2000). On the other hand, if $N_{h} / N_{v}$ is small, we can write

$$
\Lambda_{h}=b_{h} \beta_{h v} \frac{I_{v}}{N_{v}}, \quad \Lambda_{v}=b_{v} \beta_{v h}\left(\frac{I_{h}}{N_{h}}+\widetilde{\beta_{h v}} \frac{R_{h}}{N_{h}}\right),
$$

where $b_{h}\left(N_{h}, N_{v}\right) \approx \sigma_{h}$ and $b_{v}\left(N_{h}, N_{v}\right) \approx N_{h} \sigma_{h} / N_{v}$, which corresponds to the original mass action model of Ross, that was written in terms of fractions of the population, [5], [3]. In particular, if the populations are constant, then this model is the usual mass action compartmental model.

Finally, we mention saturated infection rates, where the force of infection is given by a version of the Holling II functional response. It was first introduced to infectious disease modelling in [19] in their study of the cholera epidemic. We can adopt such a force of infection for malaria models with treatment or acquired immunity. Then, assuming that only individuals from the infected class $I_{h}$ are infectious, the force of infection of humans by mosquitoes and the force of infection of mosquitoes will be given by, respectively,

$$
\Lambda_{h}=\sigma_{v} \beta_{h v} \frac{I_{v}}{1+\eta_{v} I_{v}}, \quad \Lambda_{v}=\sigma_{v} \beta_{v h} \frac{I_{h}}{1+\eta_{h} I_{h}},
$$

with an obvious modification if also $R_{h}$ individuals can contribute to the infections.

The use of the Holling type II incidence function to model the infection process reflects the fact that the number of effective contacts between infective and susceptible individuals may saturate at high infective levels due to overcrowding, or due to the preventive measures applied in response to the disease, [42], [49], [58], [68], [81], [59].

\section{B. Choice of the demographic functions}

The demographic terms $f_{h}\left(N_{h}\right)$ and $g_{h}\left(N_{h}\right)$ can take several forms, depending on the population, and we list the common ones. We note that similar choices work for both $f_{v}\left(N_{v}\right)$ and $g_{v}\left(N_{v}\right)$. We also assume that there is no vertical transmission of the infection, so all new newborns are susceptible.

1) There are births with a constant total birth rate $\Pi_{h}$ and natural deaths with per capita natural death rate $\mu_{h}$ so that $f_{h}\left(N_{h}\right)=\Pi_{h}$ and $g_{h}\left(N_{h}\right)=\mu_{h}$. In this case, the vital dynamics (dynamics in the absence of infection) will be $\frac{d N_{h}}{d t}=\Pi_{h}-\mu_{h} N_{h}$. We note that this choice is used mostly for mathematical convenience as fitting this model to real populations produces unrealistic rates $\Pi_{h}$ and $\mu_{h}$, [52].

2) The demography is governed by the Malthusian law, that is, the total birth and death rates are proportional to the total population, [20], [57]. Thus, $f_{h}\left(N_{h}\right)=\psi_{h} N_{h}$ and $g_{h}\left(N_{h}\right)=\mu_{h}$ and in this case the vital dynamics is given by $\frac{d N_{h}}{d t}=\left(\psi_{h}-\mu_{h}\right) N_{h}$.

3) The birth rate is directly proportional to the total human population, $f_{h}\left(N_{h}\right)=\psi_{h} N_{h}$, while the death rate depends on the density of the population size in a nonlinear way. For instance, in addition to the intrinsic deaths, there may be additional deaths due to the overcrowding, which can be modelled as $g_{h}\left(N_{h}\right)=\mu_{h}+\widetilde{\mu_{h}} N_{h}$, where $\widetilde{\mu_{h}}$ is 




(a) Line plot of number of indigenous reported malaria cases

Figure 2: Line plots of the number of indigenous reported malaria cases and deaths in South Africa for the years $2001-2018$.

the additional constant death rate. Then the vital dynamics is given by $\frac{d N_{h}}{d t}=\psi_{h} N_{h}-$ $N_{h}\left(\mu_{h}+\widetilde{\mu_{h}} N_{h}\right)$, where we assume that $\psi_{h}$ is sufficiently large so that the vital dynamics is logistic.

We can use other types of birth functions, such as the Ricker function, [65], the BevertonHolt function, [13] or the Maynard-Smith-Slatkin function, [72].

C. Fitting the malaria model into the South African data

According to the WHO report 2019 [78], South Africa reported 9540 indigenous cases of malaria in 2018, with 69 deaths. However, the number of reported indigenous cases of malaria in 2017 was 22 064. In South Africa, malaria is transmitted mainly in the border areas due to the crossborder movement of populations, including workers from neighbouring malaria-endemic countries and South Africa residents travelling there. We also note here that according to the Department of Health of the Republic of South Africa, some parts of the country are endemic for malaria. At the same time, $10 \%$ of the population (approximately 4.9 million people) is at risk of contracting the

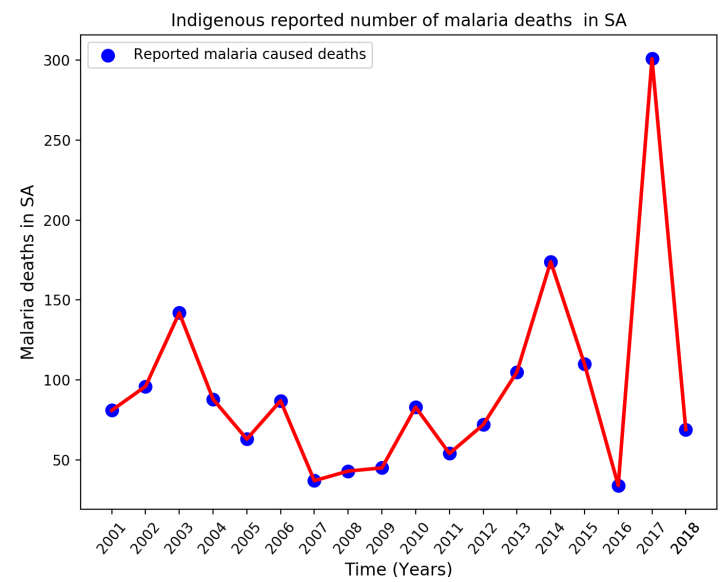

(b) Line plot of number of indigenous reported malaria caused deaths disease, with $P$. falciparum being the dominant malaria species.

A summary of the number of reported indigenous malaria cases and deaths in South Africa for the years 2001-2018 is shown in Figure 2. We plotted them in the Python programming language using data from the WHO website, [79].

Figure 3 and Figure 4 depict curves fitted from model (1) using malaria data obtained from (WHO-GHO, 2016) for the number of indigenous reported malaria cases and malaria caused deaths, respectively, in South Africa in $2001-2018$. Here we use the forces of infections $\Lambda_{h}=b_{h} \beta_{h v} \frac{I_{v}}{N_{v}}$ and $\Lambda_{v}=b_{v} \beta_{v h}\left(\frac{I_{h}+\zeta_{r} R_{h}+\zeta e E_{h}}{N_{h}}\right)$. We set the initial conditions $S_{h}(2001)=5 \times 10^{6}, R_{h}(2001)=0$ and let $I_{h}(2001)=$ to vary between 15,000 and 30,000 , since in 2001 the number of indigenous reported malaria cases was 26506. We then assumed $E_{h}(2001)=50000, S_{v}(2001)=50000$, $E_{v}(2001)=5000$ and $I_{v}(2001)=4000$. Furthermore, as a guessed starting parameters we used the baseline values listed in Table 1, obtained from literature except for the population projection of South Africa in (Worldometers, 2020), where we estimated $\mu_{h}=0.0159$ per year since the average 
lifespan of South Africans in 2017 was 63 years and $\Pi_{h}=70000$ per year since the annually approximately $7 \times 10^{5}$ people are added to the total population but only $10 \%$ are at risk of malaria. We use $\mu_{v}=17.38$ per year since the lifespan of an Anopheles mosquito is 10-21 days.

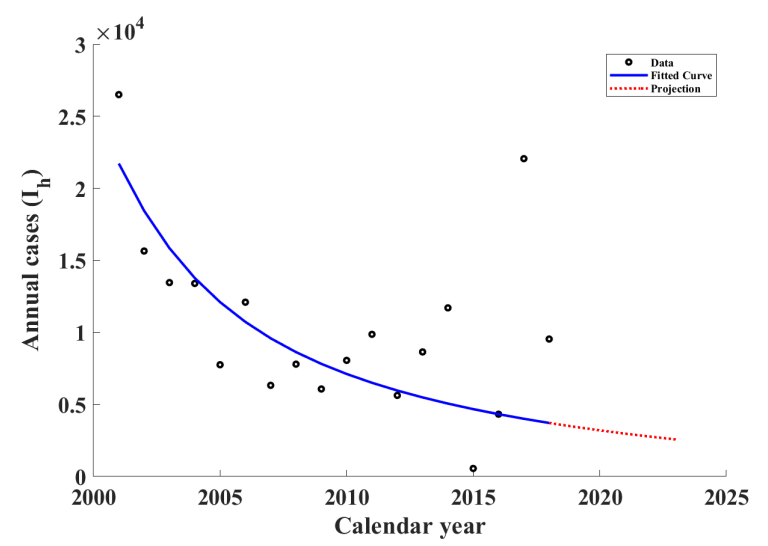

Figure 3: A fitted curve for indigenous reported malaria cases in South Africa , $I_{h}$ for model (1) using data from WHO-GHO [79] for the years $2001-2018$, and projections up to 2025 .

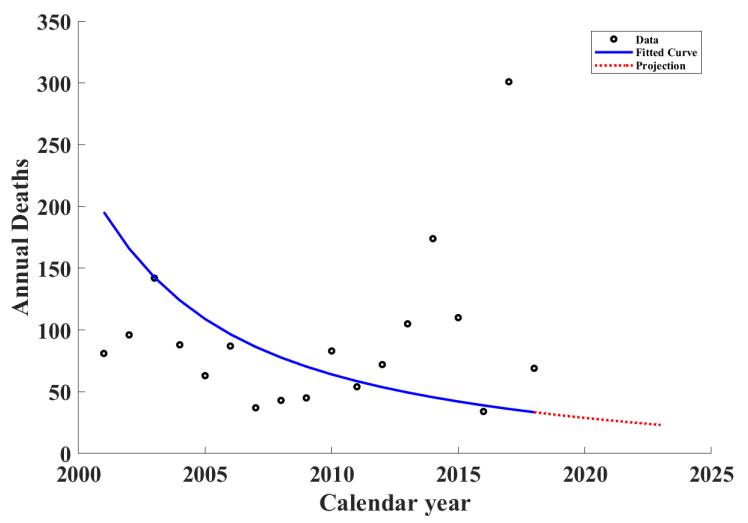

Figure 4: A fitted curve for indigenous reported malaria caused deaths in South Africa, for the model (1) using data from [79] for the years 20012018 , and projections up to 2025 . The method used to fit the model in both cases is lsqcurvefit with multi-start for global fit in MATLAB.

We observe that the theoretical curves in Figures 3 and 4 do not cater well for short term variations in the number of cases and deaths. This is since neither model considers seasonal or environmental variations that heavily affect malaria incidence. In particular, the spike in 2017 could be partly attributed to the higher than normal rainfall between the 2015 - 2016 and subsequent drought in Southern Africa.

Parameter uncertainty and the predicted uncertainty is important for qualifying a confidence in the solution, and for adjusting parameter values so that a correlation best fits data. As it is directly mentioned in here, "the $95 \%$ confidence bands enclose the area that you can be $95 \%$ sure contains the true curve. It gives you a visual sense of how well your data define the best-fit curve. As such, we extend the data fitting in Figures 3 and 4 to include a $95 \%$ confidence bands, as can be observed in Figure 5 and Figure 6.

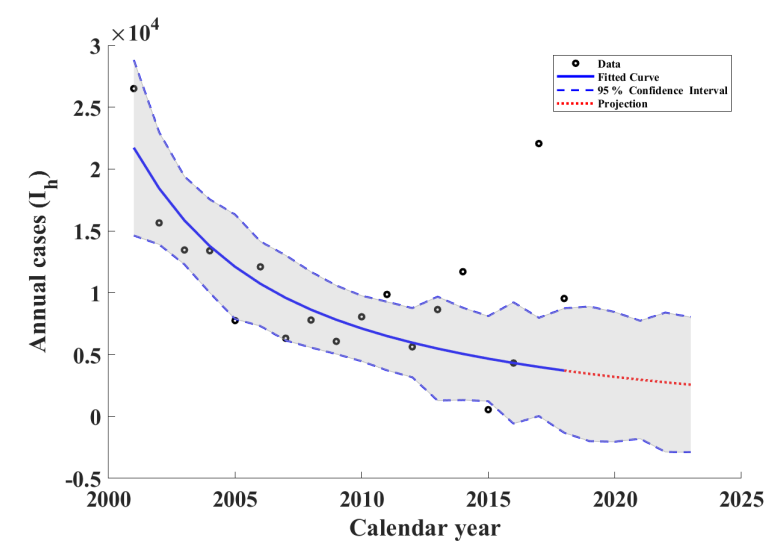

Figure 5: A fitted curve for indigenous reported malaria cases in South Africa showing a 95\% Confidence interval, for the model (1) using data from (WHO-GHO, 2016) for the years 2001-2018, and projections up to 2025 .

We note here that while fitting model (1) to the data using the baseline values as guessed starting parameter values with their corresponding lower and upper bounds in Table I. we only let six parameters, namely, $C_{h}, C_{h}, v_{h}, v_{v}$ and $\rho_{h}$ to be unknown so that the algorithm will estimate their values with a $95 \%$ confidence interval.

As can be seen in Figure 5 or Figure 6, several of data points lie below and above the fitted 
J Banasiak, R Ouifki, W A Woldegerima, Some mathematical tools for modelling malaria: a subjective ...

Table I: Parameters, their baseline values used for fitting, dimension, references and lower and upper bounds used. Several of the ranges for the parameter values are directly taken from [21], where they used data for the high and low transmission areas. Other parameter ranges are adapted from [17], [33] and some are estimated.

\begin{tabular}{|l|l|l|l|l|}
\hline Parameter & $\begin{array}{l}\text { Baseline } \\
\text { Value Used }\end{array}$ & Dimension & Reference & $\begin{array}{l}\text { Ranges (lower \& upper } \\
\text { bounds used) }\end{array}$ \\
\hline$\Pi_{h}$ & 70000 & $H \times$ ear $^{-1}$ & Calculated from [82] & fixed \\
\hline$\Pi_{v}$ & 5000 & $V \times$ ear $^{-1}$ & Assumed & {$\left[1 \times 10^{3}, 5 \times 10^{5}\right]$} \\
\hline$C_{v}=\beta_{v h} b$ & 0.2 & $H \times V^{-1} \times$ ear $^{-1}$ & {$[21]$} & {$[0.01,2]$} \\
\hline$C_{h}=\beta_{h v} b$ & 0.6 & $H \times V^{-1} \times$ ear $^{-1}$ & {$[21]$} & {$[0.01,2]$} \\
\hline$v_{h}$ & 0.1 & earr $^{-1}$ & {$[21]$ ) } & {$[0,10]$} \\
\hline$\delta_{h}$ & 0.008 & year $^{-1}$ & Calculated from [79] & {$[0,0.1]$} \\
\hline$\rho_{h}$ & 0.47 & year $^{-1}$ & {$[21]$} & {$[0.0005,2]$} \\
\hline$\gamma_{h}$ & 0.275 & earr $^{-1}$ & Assumed & {$[0,10]$} \\
\hline$\zeta_{e}$ & 0.005 & 1 & Assumed & {$[0,1]$} \\
\hline$\zeta_{r}$ & 0.001 & 1 & Assumed & {$[0,1]$} \\
\hline$v_{v}$ & 0.083 & year $^{-1}$ & {$[21]$} & {$[0.005,2]$} \\
\hline$\mu_{v}$ & 17.38 & year & fixed \\
\hline$\mu_{h}$ & 0.0159 & year & fixed \\
\hline
\end{tabular}

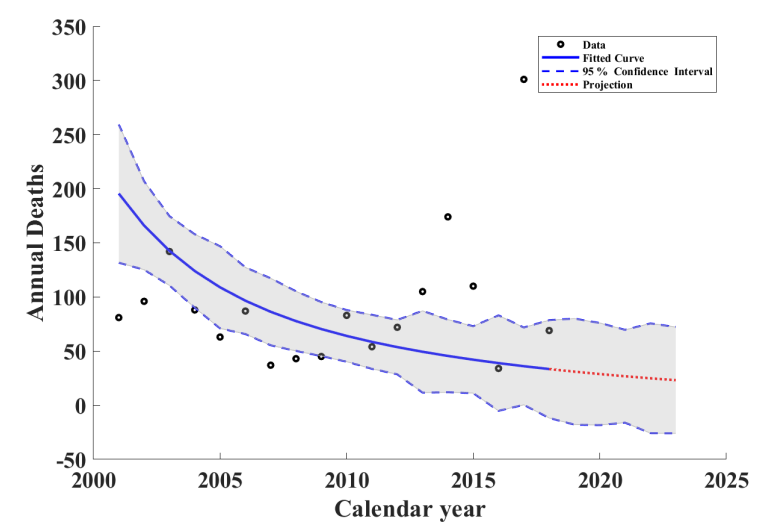

Figure 6: A fitted curve for indigenous reported malaria caused deaths in South Africa showing a 95\% confidence interval, for the model (1) for the years 2001-2018, and projections up to 2025 . To fit the model into the malaria data we used a method in MATLAB called "Isqcurvefit" (leastsquare curve fit) with multi-start for global fit, and to obtain the confidence interval we used the method called "nlpredci", which stands for a nonlinear prediction. It is a nonlinear regression prediction for confidence intervals.

lines, and some of them are outside of the $\% 95$ confidence brand. Clearly these residuals are not well-behaved and the residuals are not evenly distributed over the time series. So, we must emphasize here that the parameters of the model are not identifiable with best estimates, and they have large error ranges. This is because the model has 7 state variables and 15 parameters, but it is fitted to a time series of two observations, and thus it is overparameterized. Its proper calibration and providing relevant statistical information such as confidence interval, margins of errors and parameter estimates require more data, not available at present. Thus, we do not claim that the data fitting given in Figure 3 and Figure 4 or Figure 5 and Figure 6 are definitive. Others may argue that it could have been better to use a much simpler model or simply to use an exponential decay function to statistically fit the given data for the number of malarai cases and deaths, and estimate the fewer parameters involved in defining an exponential decay function with best parameter fit and narrow confidence interval. However, we want to use a mechanistic model, as these model when correctly constructed and validated can have a strong predictive power, and can be used to test effectiveness of an intervention, as such interventions can in principle be include mechanistically in the model.

The preceding discussion shows how impor- 
tant it is to validate the results obtained from mathematical models by comparing them with the field data. Correctly done, statistical analysis of the results allows us to assess the model's ability to simulate essential features of the transmission dynamics of malaria, that is, to validate the model. It also provides for extrapolation and interpolation of the data for future predictions and estimates of input parameters for more complex mathematical models [22], [46]. It is worth noting that though complex mechanistic models have more predictive power, the uncertainty in the parameters estimates strongly curtails this power. Thus, an important part of modelling is balancing the model's complexity with the availability of reliable data.

\section{REducing COMPLEXITY OF MODELS By MULTIPLE} SCALE ANALYSIS

One of the ways of reducing the complexity of a model is exploiting its structural features to aggregate functionally similar variables and thus simplify the model without losing salient features of is dynamics. There are various ways of models' aggregation, [8], the most popular being the one based on the existence of multiple time scales in it. Let us explain this approach using a malaria model as an example.

We have observed that, due to the interplay of host and vector dynamics as well as the complex evolution of the vector population itself, see, e.g. [58], malaria models have indeed become increasingly involved, making their robust analysis difficult if not impossible. Fortunately, biological phenomena often occur on time or size scales of widely different orders of magnitude. For instance, in malaria models, mosquitoes' vital dynamics occurs on a much faster time scale (average lifespan of fewer than 21 days) than that of humans (average lifespan of around 65 years). Because of this, malaria models can be considered as multi scale models, see, e.g. [30], which paves the way for their significant simplifications. Showing that it is indeed possible and that the simplified models preserve essential features of the original dynamics requires a delicate mathematical analysis belonging to the field of singular perturbation theory, see, e.g. [9], [10], [66]. We shall illustrate this approach on the model given by (1) with $f_{h}\left(N_{h}\right)=\psi_{h} N_{h}, g_{h}\left(N_{h}\right)=\mu_{h} N_{h}, f_{v}\left(N_{v}\right)=\psi_{v} N_{v}$, and $g_{v}\left(N_{v}\right)=\psi_{v} N_{v}$, that is, assuming a stable mosquito population. Further, for computational simplicity, we discard the exposed classes so that

$$
\begin{aligned}
& \frac{d S_{h}}{d t}=\left(\psi_{h}-\mu_{h}\right) S_{h}+\left(\psi_{h}+\rho_{h}\right) R_{h}+\psi_{h} I_{h} \\
&-\sigma_{v} \beta_{h v} \frac{I_{v}}{S_{h}+I_{h}+R_{h}} S_{h}, \\
& \frac{d I_{h}}{d t}= \sigma_{v} \beta_{h v} \frac{I_{v}}{S_{h}+I_{h}+R_{h}} S_{h}-\left(\delta_{h}+\gamma_{h}+\mu_{h}\right) I_{h}, \\
& \frac{d R_{h}}{d t}=\gamma_{h} I_{h}-\left(\rho_{h}+\mu_{h}\right) R_{h}, \\
& \frac{d S_{v}}{d t}=\mu_{v} I_{v}-\sigma_{v} \beta_{v h} \frac{I_{h}}{S_{h}+I_{h}+R_{h}} S_{v}, \\
& \frac{d I_{v}}{d t}=\sigma_{v} \beta_{v h} \frac{I_{h}}{S_{h}+I_{h}+R_{h}} S_{v}-\mu_{v} I_{v},
\end{aligned}
$$

where we used $N_{h}=S_{h}+I_{h}+R_{h}$ and $N_{v}=S_{v}+I_{v}$.

Next, using parameter values directly from [20, Table 4.1] in a time scale of years, we write system (8) as

$$
\begin{aligned}
& \frac{d S_{h}}{d t}= 1.26 \times 10^{-2} S_{h}+5.36 R_{h}+2.8 \times 10^{-2} I_{h} \\
&-4.38 \frac{I_{v}}{S_{h}+I_{h}+R_{h}} S_{h}, \\
& \frac{d I_{h}}{d t}= 4.38 \frac{I_{v}}{S_{h}+I_{h}+R_{h}} S_{h}-1.37 I_{h}, \\
& \frac{d R_{h}}{d t}= 1.35 I_{h}-5.34 R_{h}, \\
& 10^{-3} \frac{d S_{v}}{d t}=5.22 \times 10^{-2} I_{v}-1.82 \times 10^{-1} \frac{I_{h}}{S_{h}+I_{h}+R_{h}} S_{v} \\
& 10^{-3} \frac{d I_{v}}{d t}=1.82 \times 10^{-1} \frac{I_{h}}{S_{h}+I_{h}+R_{h}} S_{v}-5.22 \times 10^{-2} I_{v},
\end{aligned}
$$

with initial condition

$$
\left(S_{h}(0), I_{h}(0), R_{h}(0), S_{v}(0), I_{v}(0)\right)=\left(S_{h}^{0}, I_{h}^{0}, R_{h}^{0}, S_{v}^{0}, I_{v}^{0}\right)
$$

We can observe that $\mu_{v}=5.22 \times 10^{1}$ per year, whereas $\mu_{h}=1.58 \times 10^{-2}$ per year, that is, they differ by 3 orders of magnitude. The idea here is to replace the factor $10^{-3}$ by a small parameter $\epsilon$ and try to approximate (9) by solutions of the 


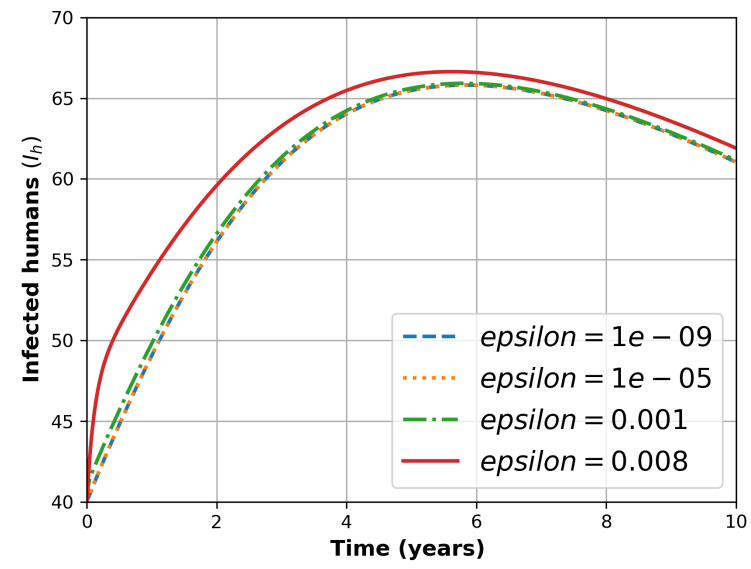

(a) infected humans

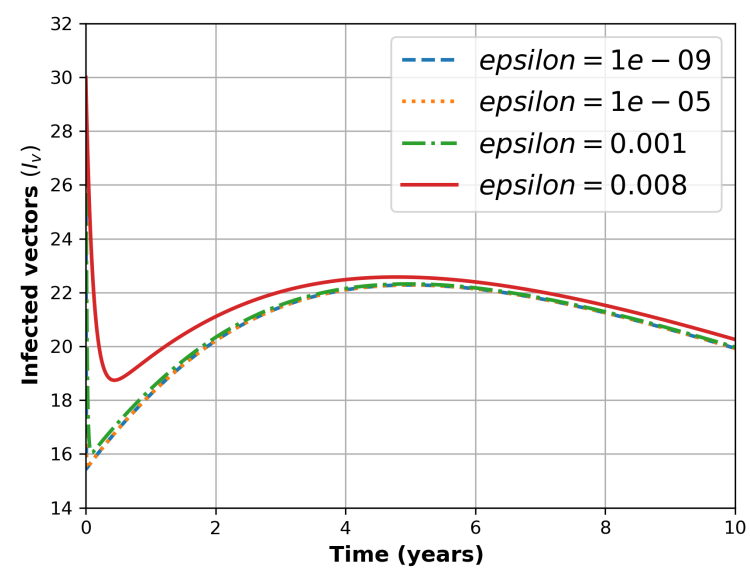

(b) infected mosquitoes

Figure 7: Solution curves for the infected class for different values of $\epsilon$. The solution of the original system corresponds to $\epsilon=10^{-3}$ and solution to 10 corresponds to $\epsilon=0$.

simplified problem with $\epsilon=0$, that is,

$$
\begin{aligned}
\frac{d \widetilde{S_{h}}}{d t}= & 1.26 \times 10^{-2} \widetilde{S_{h}}+5.36 \widetilde{R_{h}}+2.8 \times 10^{-2} \widetilde{I_{h}} \\
& -4.38 \frac{\widetilde{I_{v}}}{\widetilde{N_{h}}} \widetilde{S_{h}}, \\
\frac{d \widetilde{I_{h}}}{d t}= & 4.38 \frac{\widetilde{I_{v}}}{\widetilde{N_{h}}} \widetilde{S_{h}}-1.37 \widetilde{I_{h}}, \\
\frac{d \widetilde{R_{h}}}{d t}= & 1.35 \widetilde{I_{h}}-5.34 \widetilde{R_{h}}, \\
0= & 5.22 \times 10^{-2}\left(N_{v}-\widetilde{S_{v}}\right)-1.82 \times 10^{-1} \frac{\widetilde{I_{h}}}{\widetilde{N_{h}}} \widetilde{S_{v}},
\end{aligned}
$$

with the same initial conditions, where we used the fact that $N_{v}$ is constant.

Clearly, 10 is a lower-dimensional system and it follows that (subject to adding an initial layer corrector) its solutions approximate the solutions of (10), see [9], [10], [66]. We illustrate this result in Figure 7, where we present numerical simulations of 10 with initial conditions $S_{h}^{0}=$ $1000, R_{h}^{0}=0, I_{h}^{0}=40, S_{v}^{0}=100, I_{v}^{0}=30$, [20], and with $10^{-3}$ replaced by different values of $\epsilon$, so that with $\epsilon=10^{-3}$ we recover the solutions of system (8). The solution for (10) corresponds to $\epsilon=0$. We observe that as $\epsilon$ approaches to zero, the solutions get closer to the solution of 10 . See, Figure 7

\section{Current research towards malaria ELIMINATION AND ERADICATION}

Even though antimalarial drugs are widely available and can be used at different cycles within a human, it is unlikely that they can eliminate malaria on their own and novel strategies are urgently needed. One of them is developing interventions to interrupt or completely block the malaria transmission by targeting the transmission of either the gametocytes to the vector or the sporozoites to humans. Such transmission-blocking interventions (TBIs) can be either transmission-blocking drugs (TBDs) or transmission-blocking vaccines (TBVs), [25], [6]. Then, [74], [70], TBDs can be classified as drugs targeting the malaria parasite within the human host, drugs targeting the parasite in the vector or drugs targeting the vector itself.

Designing a drug whose primary objective is the transmission-blocking would be a game-changer leading to comprehensive malaria elimination, [14], [18]. During the drug development process, the central focus is on the ability of the compound to achieve one of the following goals: killing the malaria parasites during the liver stage or the blood 


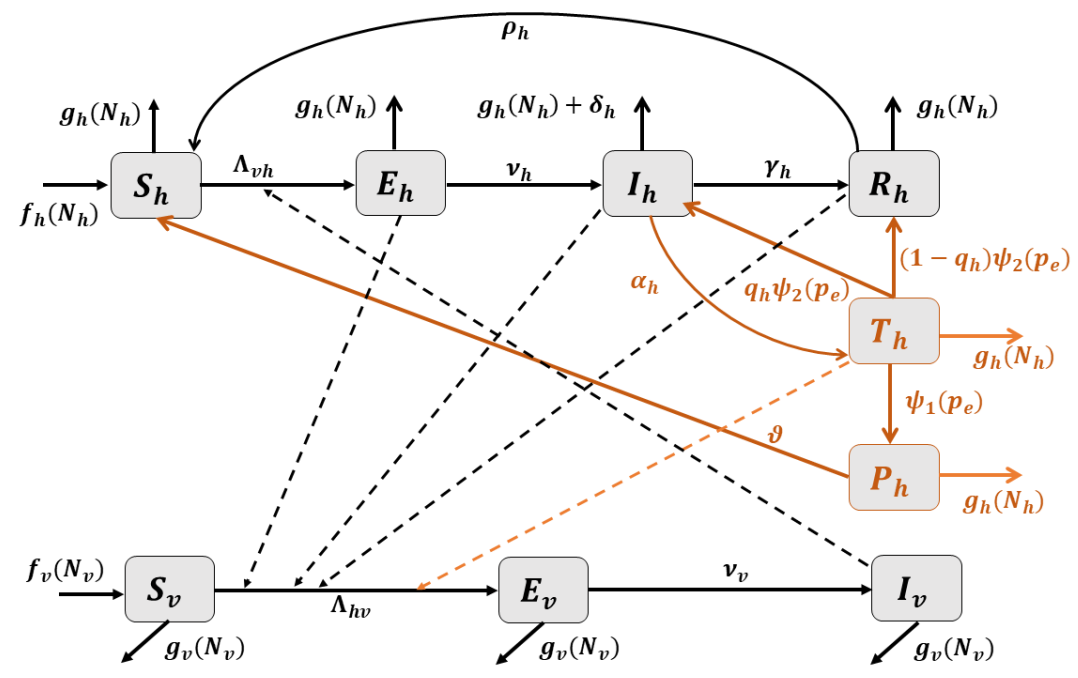

Figure 8: Flow diagram showing the malaria transmission dynamics between human and mosquito populations with transmission-blocking drug treatment.

stage of infection, blocking the formation and maturation of gametocytes, providing chemoprevention to high-risk groups or else preventing the parasite cycle within the mosquito. Mathematical models are important at this stage to guide drug development by studying its pharmacokinetics (PK) and pharmacodynamics (PD). In the first case, the models can help us study the change of the drug concentration over time as determined by its absorption, distribution, metabolism and excretion. These are typically described by a set of differential equations representing the physical compartments, where the different processes occur. In the second case, mathematical models can describe the relationship between drug concentration and its killing efficacy. This can be shown as a 'doseresponse curve' showing the efficacy as a function of the measured concentration of the drug in the blood, [38], [41], [63], [71].

Still, there is a lack of insight about the population-level impact of different strategies of rolling out the TBDs that can be obtained from mathematical modelling. In our recent work, [80], we have addressed this problem by proposing and providing a preliminary analysis of a population level mathematical model of human-mosquito in- teractions that take into account an intervention using TBDs. Our model extends (1) by including the class $T_{h}$ of people under treatment with a TBD, and the class $P_{h}$ of people who have been successfully treated and are (at least temporarily) immune to malaria and (at least temporarily) does not transmit malaria. We allow the treatment to fail in which case the individuals from $T_{h}$ move back to $I_{h}$ or $R_{h}$ (so that they remain infectious) or be successful whereby the individuals stop being infections. The model can be represented by the flow diagram in Figure 8.

Our model is still preliminary and, in particular, it has not been tested on the real data that, due to the novelty of the field, are hard to obtain. Nevertheless, we determined a threshold quantity that governs the spread of malaria under treatment by a TBD and, using this threshold, we investigated the impact and sensitivity of the model parameters that can be manipulated to drive the threshold quantity below unity. We also derived an expression that relates the drug efficacy and its treatment rate, and determined a critical treatment coverage rate, that is, the rate beyond which the TBD can eliminate malaria.

On the other hand, also research for a malaria 
vaccine has been underway. In particular, significant progress has been made in this field with the development of the pre-erythrocytic vaccine, named RTS,S. However, the use of malaria vaccines is not yet implemented well.

As noted before, a limitation of such complex malaria models is that they can be overparameterized and it could be impossible to fit the parameters accurately on a given data set. This creates uncertainty in the simulation results. The main problem is that we require extensive data sets for estimating the parameter values with small error margins, as models of malaria are usually highly complex.

\section{Conclusion}

Mathematical modelling is a process that uses mathematical tools to represent, analyze, make predictions, or otherwise provide insight into realworld phenomena. The value of mathematical models lies in that they help us to understand realworld phenomena by simplifying complex scenarios. For instance, analysis of epidemiological models informs us about their dynamics and thus enables predictions about the development of the disease. In this way, mathematical models offer a cheap alternative to expensive, impractical, or impossible field and experimental work. Mathematical models also have their disadvantages and shortcomings. A model is just an approximation of a real-life phenomenon since the complexity of nature makes it impossible to exactly represent it by a manageable set of equations. A mathematical model is as good as the assumptions used to formulate it, and thus it will work only when these assumptions are satisfied. Even after a model is formulated and analyzed, the results may be not entirely conclusive, since the theory on which we base it may be inadequate, or the available data do not suffice for its validation. In particular, for malaria, the data availability is often not sufficient for reliable identification of the parameters, and this uncertainty strongly limits the predictive power of many models.

Nevertheless, over the past century, mathematical models of malaria of various levels of com- plexity concerning the human, mosquito, and Plasmodium parasite populations have been developed and studied to understand the malaria infection mechanisms and thus facilitate its control and, ultimately, elimination. Moreover, mathematical modelling of malaria dynamics is important to guide the drug development, suggest a deployment strategy and quantify adequate treatment coverage. Whenever possible, quantitative and qualitative results from mathematical models of malaria are compared with the observational data to identify the model's strengths and weaknesses. In many cases, however, the scarcity of reliable data on the human behaviour, the life cycles and behaviour of both the vector and the parasite, proliferation and waning of immunity, age profiles of symptomatic and asymptomatic infections or parasite's drug resistance limits the usefulness of many mathematical models in the policy-making processes. Thus, the importance of models is often not so much quantitative but rather qualitative, as was already noted by Ross and several other researchers. It is, therefore, crucial for mathematical modellers to collaborate closely with life and health scientists and public health workers to facilitate an informed and robust exchange leading to better models and knowledge-based decisions.

\section{AcKNOWLEDGEMENT}

All authors acknowledge the financial support from the DST/NRF SARChI Chair in Mathematical Models and Methods in Biosciences and Bioengineering at the University of Pretoria, grant No. 82770.

\section{CONFLict OF INTERest}

All authors declare no potential conflict of interest.

\section{REFERENCES}

[1] Allen, L. J., Brauer, F., Van den Driessche, P., and Wu, J. Mathematical epidemiology, vol. 1945. Springer, 2008.

[2] Alonso, P. L., Brown, G., Arevalo-Herrera, M., Binka, F., Chitnis, C., Collins, F., Doumbo, O. K., Greenwood, B., Hall, B. F., Levine, M. M., ET Al. A research agenda to underpin malaria eradication. PLoS Med 8, 1 (2011), e1000406. 
[3] Anderson, R. M. The population dynamics of infectious diseases: theory and applications. Springer, 2013.

[4] Anderson, R. M., Anderson, B., And May, R. M. Infectious diseases of humans: dynamics and control. Oxford university press, 1992.

[5] Anderson, R. M., and May, R. M. Population biology of infectious diseases: Part i. Nature 280, 5721 (1979), 361-367.

[6] Andrews, K. A., Wesche, D., McCarthy, J., Möhrle, J. J., Tarning, J., Phillips, L., Kern, S., and Grasela, T. Model-informed drug development for malaria therapeutics. Annual review of pharmacology and toxicology 58 (2018), 567-582.

[7] Aron, J. L., And May, R. M. The population dynamics of malaria. In The population dynamics of infectious diseases: theory and applications. Springer, 1982, pp. 139179.

[8] Auger, P., De La Parra, R. B., Poggiale, J.-C., Sánchez, E., And Nguyen-Huu, T. Aggregation of variables and applications to population dynamics. In Structured population models in biology and epidemiology. Springer, 2008, pp. 209-263.

[9] Banasiak, J., Falkiewicz, A., and Tchamga, M. S. Aggregation methods in analysis of complex multiple scale systems. In Systems Analysis Approach for Complex Global Challenges. Springer, 2018, pp. 249-276.

[10] Banasiak, J., AND Lachowicz, M. Methods of small parameter in mathematical biology. Springer, 2014.

[11] Bekiryazici, Z., Kesemen, T., And Merdan, M. Stochastic and random models of malaria disease with vertical transmission. New Trends in Mathematical Sciences 5, 1 (2017), 269-277.

[12] Beretta, E., Capasso, V., and Garao, D. G. A mathematical model for malaria transmission with asymptomatic carriers and two age groups in the human population. Mathematical biosciences 300 (2018), 87-101.

[13] Beverton, R. J., And Holt, S. J. On the dynamics of exploited fish populations, vol. 11. Springer Science \& Business Media, 2012.

[14] Birkholtz, L.-M., Coetzer, T. L., Mancama, D., Leroy, D., AND Alano, P. Discovering new transmissionblocking antimalarial compounds: challenges and opportunities. Trends in parasitology 32, 9 (2016), 669-681.

[15] Brauer, F. Mathematical epidemiology: Past, present, and future. Infectious Disease Modelling 2, 2 (2017), 113-127.

[16] Brauer, F., Castillo-Chavez, C., and Castillo-Chavez, C. Mathematical models in population biology and epidemiology, vol. 2. Springer, 2012.

[17] Bretscher, M. T., Griffin, J. T., Ghani, A. C., AND OKell, L. C. Modelling the benefits of long-acting or transmission-blocking drugs for reducing plasmodium falciparum transmission by case management or by mass treatment. Malaria journal 16, 1 (2017), 341.

[18] Burrows, J. N., Duparc, S., Gutteridge, W. E., van Huijsduijnen, R. H., Kaszubska, W., Macintyre, F., Mazzuri, S., Möhrle, J. J., and Wells, T. N. New devel- opments in anti-malarial target candidate and product profiles. Malaria journal 16, 1 (2017), 26.

[19] Capasso, V., and Serio, G. A generalization of the kermack-mckendrick deterministic epidemic model. Mathematical Biosciences 42, 1-2 (1978), 43-61.

[20] Chitnis, N., Cushing, J. M., And Hyman, J. Bifurcation analysis of a mathematical model for malaria transmission. SIAM Journal on Applied Mathematics 67, 1 (2006), 24-45.

[21] Chitnis, N., Hyman, J. M., and Cushing, J. M. Determining important parameters in the spread of malaria through the sensitivity analysis of a mathematical model. Bulletin of mathematical biology 70, 5 (2008), 1272.

[22] Cox, D. R. Principles of statistical inference. Cambridge university press, 2006.

[23] Danbaba, A., ET AL. Mathematical models and analysis for the transmission dynamics of malaria. $\mathrm{PhD}$ thesis, University of Pretoria, 2016.

[24] Danquah, B., Chirove, F., and Banasiak, J. Effective and ineffective treatment in a malaria model for humans in an endemic region. Afrika Matematika 30, 7-8 (2019), 1181-1204.

[25] Delves, M., Angrisano, F., And Blagborough, A Antimalarial transmission-blocking interventions: past, present, and future. Trends in parasitology 34, 9 (2018), 735-746.

[26] Diekmann, O., Heesterbeek, J. A. P., and Metz, J. A. On the definition and the computation of the basic reproduction ratio $\mathrm{r} 0$ in models for infectious diseases in heterogeneous populations. Journal of mathematical biology 28, 4 (1990), 365-382.

[27] Filipe, J. A., Riley, E. M., Drakeley, C. J., Sutherland, C. J., AND Ghani, A. C. Determination of the processes driving the acquisition of immunity to malaria using a mathematical transmission model. PLoS computational biology 3, 12 (2007), e255.

[28] Forouzannia, F., and Gumel, A. B. Mathematical analysis of an age-structured model for malaria transmission dynamics. Mathematical Biosciences 247 (2014), 8094.

[29] Galinski, M. R., and Barnwell, J. W. Plasmodium vivax: who cares? Malaria journal 7, 1 (2008), 1-18.

[30] Garira, W., and Mathebula, D. A coupled multiscale model to guide malaria control and elimination. Journal of theoretical biology 475 (2019), 34-59.

[31] Gething, P. W., Sмith, D. L., Patil, A. P., Tatem, A. J., Snow, R. W., AND Hay, S. I. Climate change and the global malaria recession. Nature 465, 7296 (2010), 342345.

[32] Griffin, J. T., Bhatt, S., Sinka, M. E., Gething, P. W., Lynch, M., Patoulllard, E., Shutes, E., Newman, R. D., Alonso, P., Cibulskis, R. E., et Al. Potential for reduction of burden and local elimination of malaria by reducing plasmodium falciparum malaria transmission: a mathematical modelling study. The Lancet Infectious Diseases 16, 4 (2016), 465-472. 
[33] Griffin, J. T., Ferguson, N. M., and Ghani, A. C. Estimates of the changing age-burden of plasmodium falciparum malaria disease in sub-saharan africa. Nature communications 5 (2014), 3136.

[34] Griffin, J. T., Hollingsworth, T. D., Okell, L. C., Churcher, T. S., White, M., Hinsley, W., Bousema, T., Drakeley, C. J., Ferguson, N. M., Basáñez, M.-G., et al. Reducing plasmodium falciparum malaria transmission in africa: a model-based evaluation of intervention strategies. PLoS medicine 7, 8 (2010), e1000324.

[35] Gurarie, D., and McKenzie, F. E. A stochastic model of immune-modulated malaria infection and disease in children. Mathematical biosciences 210, 2 (2007), 576597.

[36] Hamer, W. H. The Milroy Lectures on Epidemic Diseases in England: The Evidence of Variability and of Persistency of Type; Delivered Before the Royal College of Physicians of London, March 1st, 6th, and 8th, 1906. Bedford Press, 1906.

[37] Hancock, P., Thomas, M. B., and Godfray, H. An age-structured model to evaluate the potential of novel malaria-control interventions: a case study of fungal biopesticide sprays. Proceedings of the Royal Society B: Biological Sciences 276, 1654 (2009), 71-80.

[38] Hastings, I. M., and Hodel, E. M. Pharmacological considerations in the design of anti-malarial drug combination therapies-is matching half-lives enough? Malaria journal 13, 1 (2014), 62.

[39] Heesterbeek, J., And Dietz, K. The concept of ro in epidemic theory. Statistica Neerlandica 50, 1 (1996), 89-110.

[40] Heffernan, J. M., Smith, R. J., and Wahl, L. M. Perspectives on the basic reproductive ratio. Journal of the Royal Society Interface 2, 4 (2005), 281-293.

[41] Holford, N. H., AND Sheiner, L. B. Understanding the dose-effect relationship. Clinical pharmacokinetics 6, 6 (1981), 429-453.

[42] Hou, J., And Teng, Z. Continuous and impulsive vaccination of seir epidemic models with saturation incidence rates. Mathematics and Computers in Simulation 79, 10 (2009), 3038-3054.

[43] Hussein, M. I. H., Albashir, A. A. D., Elawad, O. A. M. A., AND HomeidA, A. Malaria and covid-19: unmasking their ties. Malaria Journal 19, 1 (2020), 1-10.

[44] Kermack, W. O., and McKendrick, A. G. A contribution to the mathematical theory of epidemics. Proceedings of the royal society of london. Series A, Containing papers of a mathematical and physical character 115, 772 (1927), 700-721.

[45] Koella, J. C. On the use of mathematical models of malaria transmission. Acta tropica 49, 1 (1991), 1-25.

[46] Konishi, S., AND Kitagawa, G. Information criteria and statistical modeling. Springer Science \& Business Media, 2008.

[47] Le, P. V., Kumar, P., and Ruiz, M. O. Stochastic latticebased modelling of malaria dynamics. Malaria journal 17, 1 (2018), 1-17.
[48] Li, J. Simple discrete-time malarial models. Journal of Difference Equations and Applications 19, 4 (2013), 649-666.

[49] Liv, X., AND YANG, L. Stability analysis of an seiqv epidemic model with saturated incidence rate. Nonlinear Analysis: Real World Applications 13, 6 (2012), 26712679.

[50] Macdonald, G., ET al. The epidemiology and control of malaria. The Epidemiology and Control of Malaria. (1957).

[51] Mandal, S., Sarkar, R. R., And Sinha, S. Mathematical models of malaria-a review. Malaria journal 10, 1 (2011), 1-19.

[52] Martcheva, M. An introduction to mathematical epidemiology, vol. 61. Springer, 2015.

[53] Martcheva, M., Tuncer, N., and St Mary, C. Coupling within-host and between-host infectious diseases models. Biomath 4, 2 (2015), 1510091.

[54] Мвоgo, R. W., Luboobi, L. S., and Odhiambo, J. W. A stochastic model for malaria transmission dynamics. Journal of applied mathematics 2018 (2018).

[55] Molyneux, D. H., Hopkins, D. R., And Zagaria, N. Disease eradication, elimination and control: the need for accurate and consistent usage. Trends in parasitology 20, 8 (2004), 347-351.

[56] Mukhtar, A. Y., Munyakazi, J. B., and Ouifki, R. Assessing the role of climate factors on malaria transmission dynamics in south sudan. Mathematical biosciences 310 (2019), 13-23.

[57] Ngwa, G. A., And Shu, W. S. A mathematical model for endemic malaria with variable human and mosquito populations. Mathematical and Computer Modelling 32, 7-8 (2000), 747-763.

[58] Ngwa, G. A., Тевоh-Ewungkem, M. I., Dumont, Y., OUIFKI, R., AND BANASIAK, J. On a three-stage structured model for the dynamics of malaria transmission with human treatment, adult vector demographics and one aquatic stage. Journal of theoretical biology 481 (2019), 202-222.

[59] Ngwa, G. A., Woldegerima, W. A., And TEBOHEWUNGKEM, M. I. A mathematical study of the implicit role of innate and adaptive immune responses on the within-human plasmodium falciparum parasite levels. Journal of Biological Systems 28, 02 (2020), 377-429.

[60] Okosun, K. O., Ouifki, R., and Marcus, N. Optimal control analysis of a malaria disease transmission model that includes treatment and vaccination with waning immunity. Biosystems 106, 2-3 (2011), 136-145.

[61] Okuneye, K., Abdelrazec, A., and Gumel, A. B. Mathematical analysis of a weather-driven model for the population ecology of mosquitoes. Mathematical Biosciences $\mathcal{E}$ Engineering 15, 1 (2018), 57.

[62] Parham, P. E., and Michael, E. Modeling the effects of weather and climate change on malaria transmission. Environmental health perspectives 118, 5 (2010), 620626. 
[63] Patel, K., Simpson, J. A., Batty, K. T., Zaloumis, S., AND Kirkpatrick, C. M. Modelling the time course of antimalarial parasite killing: a tour of animal and human models, translation and challenges. British journal of clinical pharmacology 79, 1 (2015), 97-107.

[64] Pemberton-Ross, Peter and Chitnis, Nakul and Pothin, Emilie and Smith, Thomas. A stochastic model for the probability of malaria extinction by mass drug administration. Malaria journal 16, 1 (2017), 1-9.

[65] Ricker, W. E. Stock and recruitment. Journal of the Fisheries Board of Canada 11, 5 (1954), 559-623.

[66] Rocha, F., Aguiar, M., Souza, M., and Stollenwerk, $\mathrm{N}$. Time-scale separation and centre manifold analysis describing vector-borne disease dynamics. International Journal of Computer Mathematics 90, 10 (2013), 21052125.

[67] Ross, R. The prevention of malaria. John Murray, 1911.

[68] RuAn, S., AND WANG, W. Dynamical behavior of an epidemic model with a nonlinear incidence rate. Journal of Differential Equations 188, 1 (2003), 135-163.

[69] Sherrard-Smith, E., Hogan, A. B., Hamlet, A., Watson, O. J., Whittaker, C., Winskill, P., Ali, F., Mohammad, A. B., Uhомоiвнi, P., Maikore, I., ET AL. The potential public health consequences of covid-19 on malaria in africa. Nature medicine 26, 9 (2020), 1411-1416.

[70] Sinden, R. E. Developing transmission-blocking strategies for malaria control. PLoS Pathogens 13, 7 (2017), e1006336.

[71] Slater, H. C., OKell, L. C., and Ghani, A. C. Mathematical modelling to guide drug development for malaria elimination. Trends in parasitology 33, 3 (2017), 175184.

[72] Smith, J. M., and Slatkin, M. The stability of predatorprey systems. Ecology 54, 2 (1973), 384-391.

[73] Van den Driessche, P., AND Watmough, J. Reproduction numbers and sub-threshold endemic equilibria for compartmental models of disease transmission. Mathemati-

[78] W.H.O. World malaria report 2019. World Health Organization (2019). cal biosciences 180, 1-2 (2002), 29-48.

[74] Wadi, I., Anvikar, A. R., Nath, M., Pillai, C. R., Sinha, A., And Valecha, N. Critical examination of approaches exploited to assess the effectiveness of transmissionblocking drugs for malaria. Future medicinal chemistry 10, 22 (2018), 2619-2639.

[75] White, L. J., Maude, R. J., Pongtavornpinyo, W., Saralamba, S., Aguas, R., Van Effelterre, T., Day, N. P., AND White, N. J. The role of simple mathematical models in malaria elimination strategy design. Malaria journal 8, 1 (2009), 212.

[76] White, N. J. Pharmacokinetic and pharmacodynamic considerations in antimalarial dose optimization. Antimicrobial agents and chemotherapy 57, 12 (2013), 57925807.

[77] W.H.O. World malaria report 2018e. https://www.who. int/malaria/publications/world-malaria-report-2018/en/. 2018. Last update: 19 November 2018.

[79] WHO-GHO. Data-malaria. https://apps.who.int/gho/ data/node.main.A1362?lang=en 2016. Last updated: 2019-02-12, Acessed: 2020-09-27.

[80] Woldegerima, W. A., Ouifki, R., and Banasiak, J. Mathematical analysis of the impact of transmission-blocking drugs on the population dynamics of malaria. Applied Mathematics and Computation 400 (2021), 126005.

[81] Woldegerima, W. A., Teboh-Ewungkem, M. I., And NGwA, G. A. The impact of recruitment on the dynamics of an immune-suppressed within-human-host model of the plasmodium falciparum parasite. Bulletin of mathematical biology 81, 11 (2019), 4564-4619.

[82] WORLDOMETERS. South africa population. https://www.worldometers.info/world-population/ south-africa-population/, 2020.

[83] Yang, H. M., and Ferreira, M. U. Assessing the effects of global warming and local social and economic conditions on the malaria transmission. Revista de saude publica 34 (2000), 214-222. 